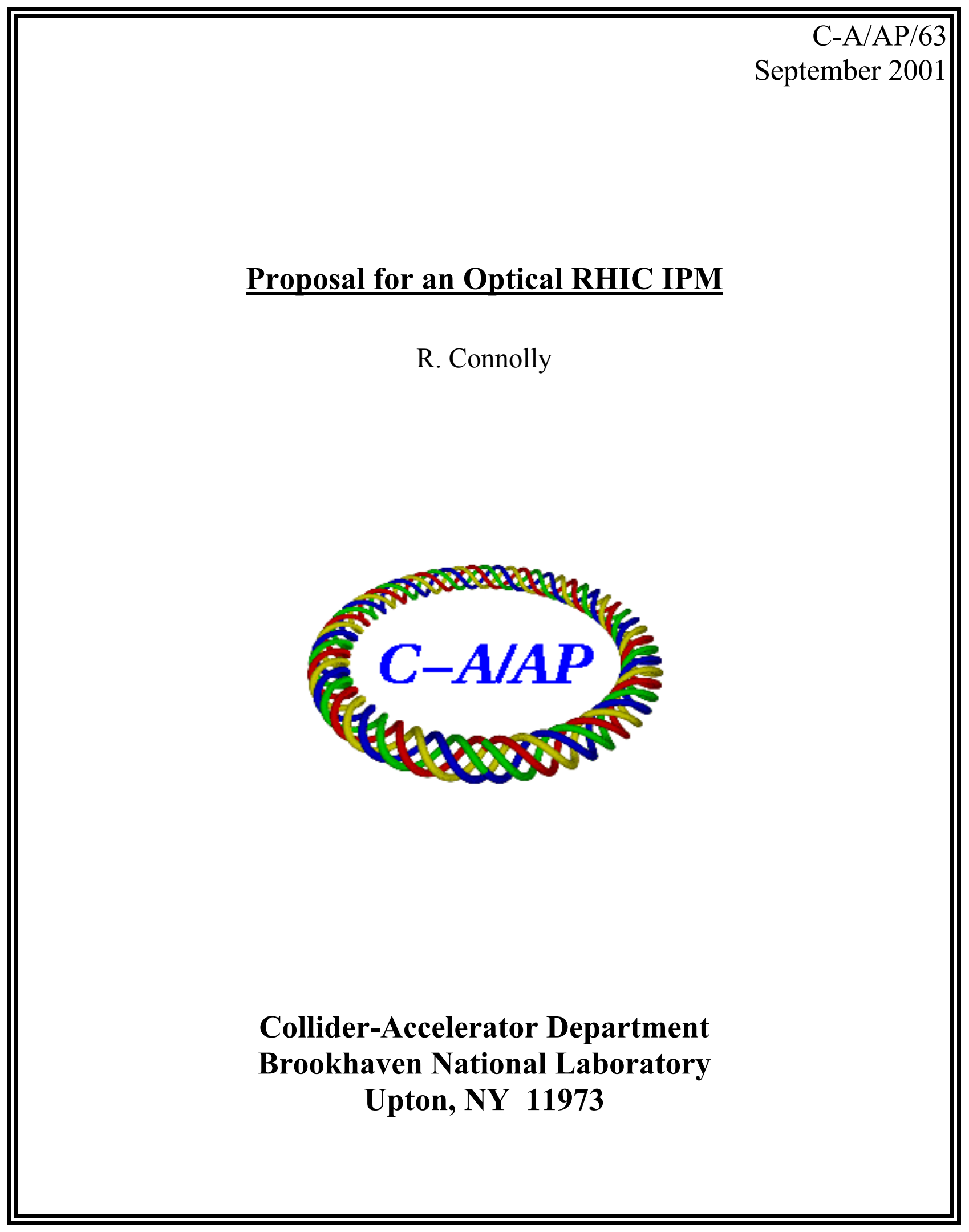




\title{
Proposal for an Optical RHIC IPM
}

\author{
Roger Connolly
}

\section{Overview of IPM problems}

\section{Beam-induced noise}

The ionization beam profile monitor in RHIC is designed to measure profiles of individual bunches with 120 bunches in the ring (three 28-MHz bucket separation) [1]. The IPM collects electrons generated from beam ionization of background gas. Each time a beam bunch passes through the detector chamber a pulse of electrons with the spatial distribution of the beam is swept from the pipe, amplified by a microchannel plate (MCP) and collected on 64 detector channels. The charge collected on each channel is amplified by a charge-sensitive amplifier and shaper amplifier which puts out a 100-ns pulse.

During the run of 1999 and early in the runs of 2000 and 2001 the detectors worked well. The only problems encountered were a few dead channels. However during the 2000 run, as the beam intensity in RHIC increased, the detectors developed a beam-induced ringing which grew until the noise pickup saturated the amplifiers. The cause of this ringing was found and detector shielding was developed which eliminated ringing in a pulsed-wire measurement intended to simulate the beam. Shielding was installed on one detector at the end of the run and beam-induced noise was not seen.

In 2001 the IPM gave good profile data early in the run but as the beam intensity increased the beam once again started to cause noise which made the profile signal from the electrons useless. The fix in 2001 is to use low-pass-filtered amplifier outputs which were available on the amplifiers. These give noise-free data but the profiles have an integration time constant of $1.6 \mu$ s so the pulses are an average of about $20 \%$ of the ring. We have lost the capability to measure individual bunch profiles.

\section{MCP characteristics}

A microchannel plate is a $1 \mathrm{~mm}$-thick sheet of glass with a honeycomb pattern of $25 \mu$ m-diameter holes. Each of the two MCP's in a RHIC IPM has $7.9 \times 10^{6}$ channels. The total glass surface area per MCP is one $\mathrm{m}^{2}$. The length/diameter ratio of a channel is 40 . An MCP is compatible with a high-vacuum environment but it requires a long pumpdown period.

When the detector was removed in 2000 to install EMI shielding and replaced without an offline bakeout it was not usable for several weeks. Even after the detector chamber vacuum had recovered it was not possible to operate the detector. When bias voltage was applied electron cascades through the channels would liberate adsorbed gases and cause a vacuum runaway.

MCP's have a lifetime of about $0.1 \mathrm{C} / \mathrm{cm}^{2}$. If the bias supplies are turned on for $1 \mathrm{~s}$ each time a measurement is made, then the plates will show signs of gain depletion after approximately $10^{5}$ measurements. Since the charge delivery is not uniform across the 
MCP the gain drop is not uniform. Lower gains in the center of the beam signal will cause artificially large beam-width measurements.

\section{Optical detection of electrons}

\section{Concept}

In the present detector the signal is electric charge which passes through the vacuum envelope. From operational experience it is clear that the electron signal from the beam provides an accurate and fast measure of beam profiles. However the collector channels also are antennas which couple to the beam fields. Electrical detection of the electrons requires a window which is transparent to electrons and opaque to EMI. This is a problem that has not been solved.

At DESY and at CERN beam profiles have been measured by detecting the ionized electrons optically with phosphor screens $[2,3]$. The usual method is to place a MCP and phosphor screen in the vacuum and view the screen with a CCD camera through a vacuum window. Recently CERN has tested a system which images the phosphor screen onto a multi-anode photomultiplier tube (PMT).

I propose that we investigate using optical detection in the RHIC IPM's. Passing the electron signal through the vacuum envelope optically should eliminate the EMI coupling. A limited R\&D project will be required to measure both the time and space resolutions of the proposed system. If these measurements show good results the IPM's would be modified by adding conflat windows to the existing vacuum chambers and installing optical elements mounted on the existing support structures. The IPM magnets would not be changed and the existing amplifiers will be used in the new system.

\section{Detector design}

The figure shows the proposed detector design. The electrons from the beam pipe are swept from the pipe and impinge on a phosphor screen which is coated on the angled face of a fiber-optic image conduit. Light from the phosphor is transported along the $4.2 \times 19.2 \mathrm{~mm}$ rectangular fiber-optic bundle. Where the conduit clears the magnet pole tip a $45^{\circ}$ bend sends the image along a $2.75^{\prime \prime}$ conflat nipple welded to the IPM vacuum chamber. The bend, shown as an angle, will have a $100-\mathrm{mm}$ radius. The end of the optical conduit is imaged through a vacuum window onto the photocathode of a 32-anode PMT which is located out of the vacuum.

\section{Phosphor screen}

The table lists parameters of five phosphor materials which look promising for this application. The light decay time needs to be faster than 110 ns to separate bunches spaced by three rf buckets. Also the light has to be peaked around $400 \mathrm{~nm}$ since this is the maximum sensitivity of the phototube. At this time I am collecting information on light output, vacuum compatibility, radiation resistance, and lifetime for these materials. 


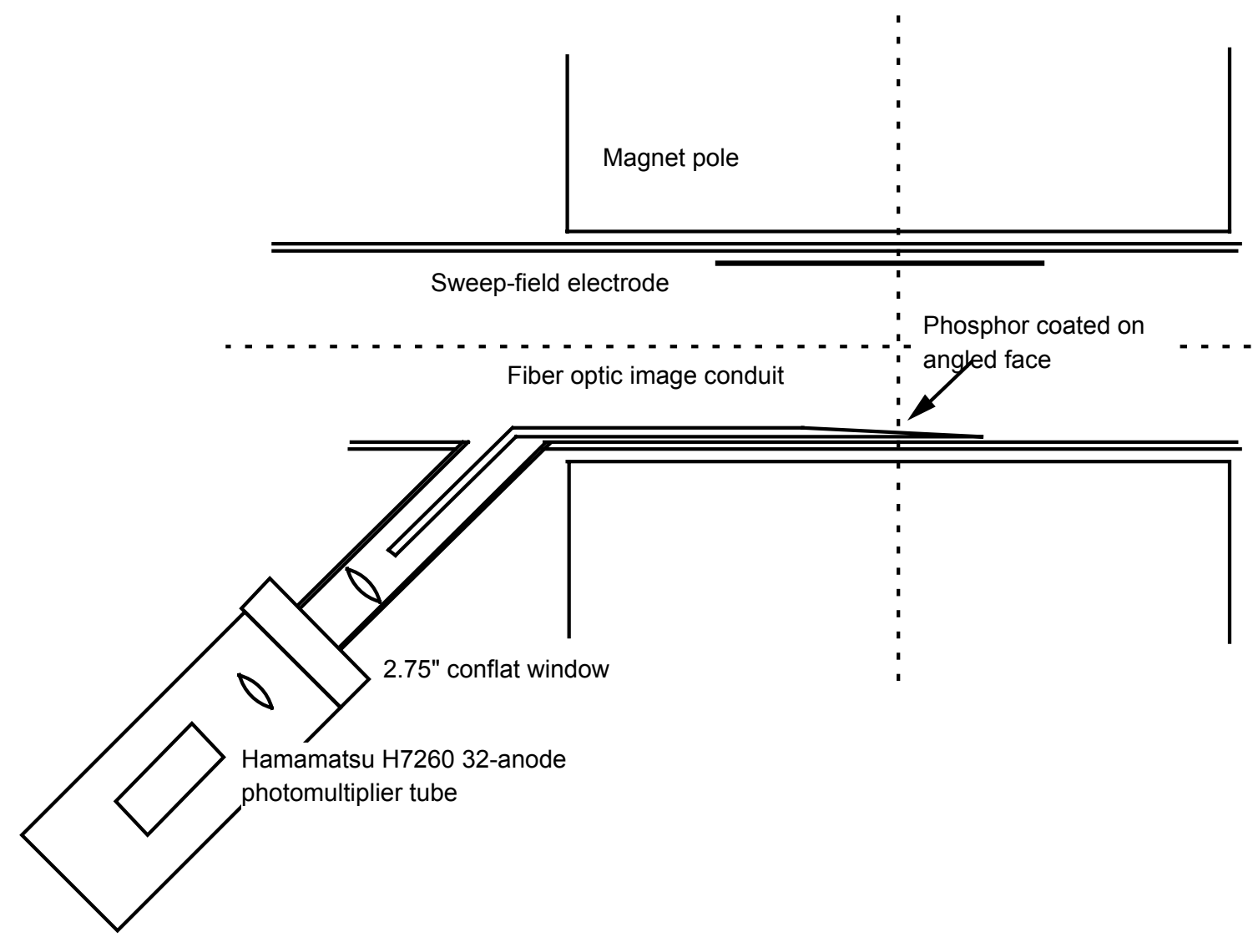

Schematic of optical detection added to existing IPM chamber and magnet. Light generated by electrons striking the angled face of the fiber optic conduit is transported to the end of the conduit and focused onto the cathode of the PMT.

Parameters of five possible phosphors.

\begin{tabular}{|l|l|l|l|l|}
\hline Phosphor & common name & color light & peak $(\mathrm{nm})$ & decay to $10 \%(\mathrm{~ns})$ \\
\hline $\mathrm{Y}_{2} \mathrm{SiO}_{5}: \mathrm{Ce}$ & P47 & blue & 400 & 100 \\
\hline $\mathrm{YAIO}_{3}: \mathrm{Ce}$ & YAP & blue & 370 & 25 \\
\hline $\begin{array}{l}\mathrm{Y}_{3} \mathrm{Al}_{5} \mathrm{O}_{12}: \mathrm{C} \\
\text { e }\end{array}$ & YAG & green & 550 & 70 \\
\hline $\mathrm{ZnO}: \mathrm{Ga}$ & & blue & 390 & 5 \\
\hline $\mathrm{C}_{4} \mathrm{H}_{10}$ & anthracene & blue & 447 & 32 \\
\hline
\end{tabular}

\section{Image detection}


The electron-generated light will be transported around the magnet pole tips with a rectangular fiber-optic image conduit. One end of the conduit will be polished at a very shallow angle to give a long face along the beam direction and the other end will be perpendicular to the conduit. The angled face will be coated with an appropriate phosphor. A two-achromatic-lens system will project the image of the end of the conduit through a window and onto the image plane of a Hamamatsu H7260 photomultiplier tube.

The H7260 PMT has an anode that is segmented into 32 channels. The channels are separated by $1 \mathrm{~mm}$ so to get $0.6 \mathrm{~mm}$ collector channels the imaging system will have a magnification of 1.67. The PMT channels are $7 \mathrm{~mm}$ tall so the optical conduit will be $7 \mathrm{~mm} / 1.67=4.2 \mathrm{~mm}$ thick. A data sheet for this tube is attached at the end of this note.

The photocathode has a quantum efficiency of $20 \%$ so one cascade should be started for each 5 photons. With a gain of $10^{6}$, one cascade will generate $35 \mathrm{fC}$ of charge at the anode. The present IPM amplifiers have a noise floor of about $5 \mathrm{fC}$. Also the PMT maximum anode current per channel is $6 \mu \mathrm{a}$. For 120 bunches in the ring this would be $640 \mathrm{fC} /$ bunch. The IPM amplifiers saturate at about $200 \mathrm{fC}$. We should be able to use the existing IPM amplifiers for the optical IPM without modification.

\section{Estimate of signal size}

Several companies make fiber optic image conduits and the available range of numerical aperture seems to be $0.55-0.65$. For the purpose of estimate I use N.A. $=0.60$. N.A. is the sine of the half angle of acceptance of an optical system. If a phosphor screen is covered with a reflective layer as is custom for vacuum installations this means that approximately $4.2 \%$ of the generated photons from electron impact will travel down the fiber.

The proposed two-achromat imaging system will have a N.A. $=0.125$. It is necessary to keep this figure at this level for accurate imaging over a large focal plane. About 8.4\% of the photons will be transported through the lens system. A $6 \mathrm{keV}$ electron produces about 200 photons in P47 and the photocathode of the PMT has a quantum efficiency of $20 \%$. Stacking up these numbers without any other loss included gives a total electron detection efficiency of the system of $1 / 7$, ie. the system will see one electron in seven that strikes the phosphor. For comparison the present system has a detection efficiency of $1 / 3$.

\section{Proposed development program}

The company, Collimated Holes, Inc., has offered to produce two prototypes using existing $5-8 \mathrm{~mm}$ optic guides for $\$ 2,500$. These would be from two different types of conduit so we could test resolutions and light coupling. Fiberguide and Burle Optics also have offered to produce a custom light guide. Applied Scintillation Technologies will coat any screen with any of the phosphors listed in the table. The Hamamatsu PMT costs about $\$ 2,500$.

I propose buying a test fiber guide with phosphor coating, building a two-lens optic system and testing the concept using the Kimball Physics electron gun we have for calibration of the electron energy detectors in the booster. If we can achieve an 
acceptable level of performance in the test then we will make a decision for a detector for RHIC. We have spare vacuum chambers and mounting hardware. A complete replacement detector can be built in the lab. Tunnel installation will involve removing the existing chambers and replacing with the new chamber.

\section{Conclusion}

The RHIC IPM's have performed poorly each year because of beam-induced noise. Experience has shown the electron signal is a good measure of beam profile. Noise problems can be reduced by increasing the charge-integration time constant, but this removes the single-bunch measurement capability. Also the microchannel plates have a finite lifetime and it will be necessary to replace them at one or two year intervals.

It may be possible to replace the existing MCP and anode collector with a multichannel optic system which will be adequately sensitive to the electron signal and immune to the EMI generated by the beam. Available phosphors are fast enough for single-bunch imaging. Unknown at this time are questions about phosphor lifetimes in the RHIC environment.

The system proposed here will use many of the RHIC IPM components including vacuum chambers, magnets, mounting hardware, amplifiers and VME digitizers.

\section{References}

[1] R. Connolly, P. Cameron, R. Michnoff and S. Tepikian, "Performance of the RHIC IPM," Proc. 2001 PAC, Chicago.

[2] K. Wittenburg, "Experience with the Residual Gas Ionization Beam Profile Monitors at the DESY Proton Accelerators," Proc. 1992 EPAC., 24.3.-28.3, Berlin, Germany and DESY HERA 92-12

[3] C. Fischer, "Results on Prototype Measurements for LHC Beam Instruments," Proc. 2001 DIPAC, Grenoble, France. 


\section{HA A ATST PHOTOMULTIPLIER TUBE ASSEMBLY H7260, H7260A}

\section{H7260 ....... -HV INPUT PIN TYPE \\ H7260A ..... -HV INPUT CABLE TYPE}

\section{FEATURES}

- Multianode 32 Channel Linear Array

- $0.8 \times 7 \mathrm{~mm}$ Anode, $1 \mathrm{~mm}$ Channel Pitch

- High Speed Response

- Low Cross-talk ............ 3 \% (Typ.)

- Built-in Voltage Divider Circuit

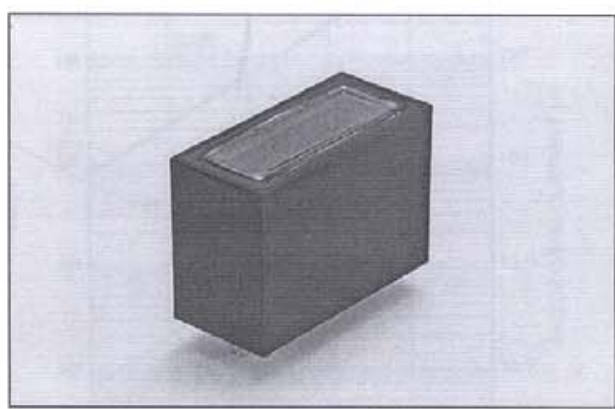

\section{GENERAL}

\begin{tabular}{|c|c|c|c|}
\hline \multicolumn{2}{|c|}{ Parameter } & Description/Value & Unit \\
\hline \multirow{2}{*}{\multicolumn{2}{|c|}{$\begin{array}{l}\text { Spectral Response } \\
\text { Wavelength of Maximum Response }\end{array}$}} & 185 to 650 & $\mathrm{~nm}$ \\
\hline & & 420 & $\mathrm{~nm}$ \\
\hline \multirow{2}{*}{ Photocathode } & Material & Bialkali & - \\
\hline & Minimum Effective Area per Channel & $0.8 \times 7$ & $\mathrm{~mm}$ \\
\hline \multicolumn{2}{|l|}{ Channel Pitch } & 1 & $\mathrm{~mm}$ \\
\hline \multicolumn{2}{|c|}{ Window Material } & UV glass & - \\
\hline \multirow{2}{*}{ Dynode } & Structure & Metal channel dynode & - \\
\hline & Number of Stages & 10 & - \\
\hline \multicolumn{2}{|l|}{ Weight } & Approx. 70 & $\mathrm{~g}$ \\
\hline \multicolumn{2}{|c|}{ Insulation Cover Material } & P.O.M. & - \\
\hline
\end{tabular}

\section{MAXIMUM RATINGS (Absolute Maximum Values)}

\begin{tabular}{|c|c|c|c|}
\hline \multicolumn{2}{|c|}{ Parameter } & \multirow{2}{*}{$\begin{array}{l}\text { Value } \\
-900\end{array}$} & \multirow{2}{*}{$\frac{\text { Unit }}{\text { Vdc }}$} \\
\hline Supply Voltage & Between Anode and Cathode & & \\
\hline Average Anode & Channel & 6 & $\mu \mathrm{A}$ \\
\hline
\end{tabular}

\section{CHARACTERISTICS (at $25^{\circ} \mathrm{C}$ )}

\begin{tabular}{|c|c|c|c|c|c|}
\hline \multicolumn{2}{|c|}{ Parameter } & Min. & Typ. & Max. & Unit \\
\hline \multirow{2}{*}{ Cathode Sensitivity } & Luminous (2856K) & 50 & 70 & - & $\mu \mathrm{A} / \mathrm{lm}$ \\
\hline & Blue (CS-5-58 filter) & 6 & 8 & - & $\mu \mathrm{A} / \mathrm{lm}-\mathrm{b}$ \\
\hline Anode Sensitivity & Luminous (2856K) & 50 & 140 & - & $\mathrm{A} / \mathrm{lm}$ \\
\hline \multicolumn{2}{|l|}{ Gain } & - & $2 \times 10^{6}$ & - & - \\
\hline \multicolumn{2}{|c|}{ Anode Dark Current per Channel (After $30 \mathrm{~min}$. storage in the darkness) } & - & 0.2 & 2 & $\mathrm{nA}$ \\
\hline \multirow{2}{*}{ Time Response } & Anode Pulse Rise Time & - & 0.6 & - & ns \\
\hline & Transit Time Spread (FWHM) & - & $0.16^{* 1}$ & - & ns \\
\hline \multicolumn{2}{|c|}{ Pulse Linearity per Channel ( $\pm 3 \%$ deviation) } & - & 0.65 & - & $\mathrm{mA}$ \\
\hline \multicolumn{2}{|l|}{ Cross-talk } & - & $3^{-2}$ & - & $\%$ \\
\hline \multicolumn{2}{|c|}{ Uniformity Between Each Anode } & - & $1: 0.8$ & - & - \\
\hline
\end{tabular}

NOTE: Voltage Divider Current $=0.33 \mathrm{~mA}$ at $-800 \mathrm{~V}$ Input.

*1 Light is irradiated to only one channel at this measurement.

"2 The ratio of signal between a channel and an average of adjacent channels, when the incident light is given to the center channel through $0.8 \times 7 \mathrm{~mm}$ aperture.

Subject to local technical requirements and regulations, availability of products included in this promotional material may vary. Please consult with our sales otflice.

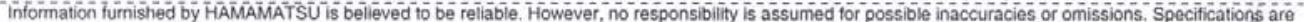
subject to change without notice. No patent rights are granted to any of the circuits described herein. 11999 Hamamatsu Photonics K.K 


\section{MULTIANODE PHOTOMULTIPLIER TUBE ASSEMBLY H7260, H7260A}

Figure 1: Typical Spectral Response

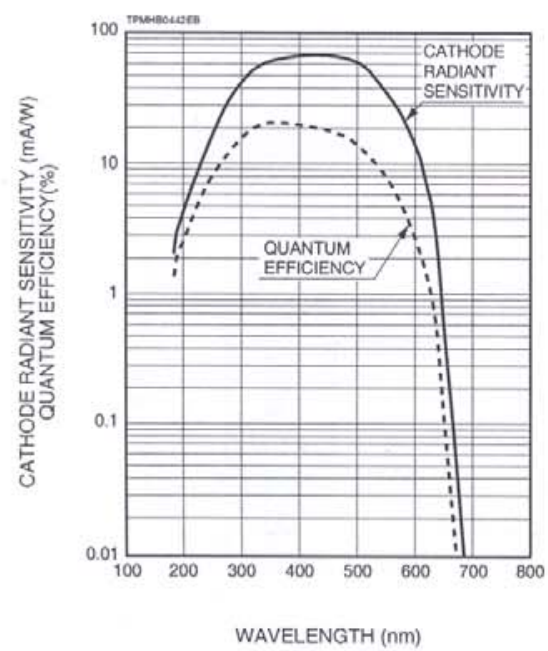

Figure 3: Typical Spatial Resolution

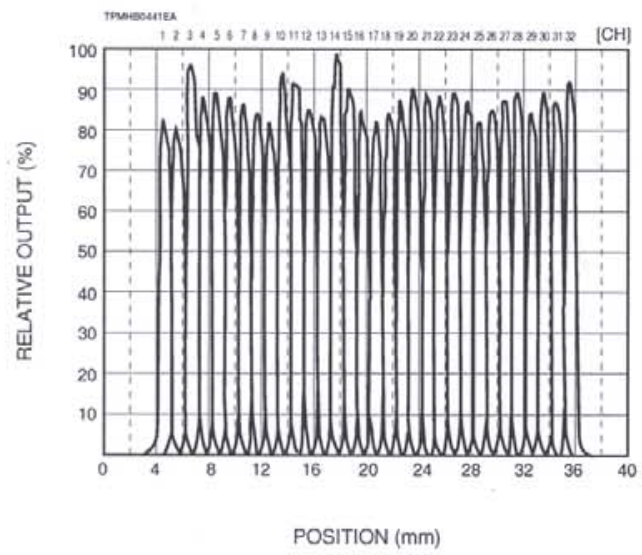

Figure 2: Typical Gain and Anode Dark Current

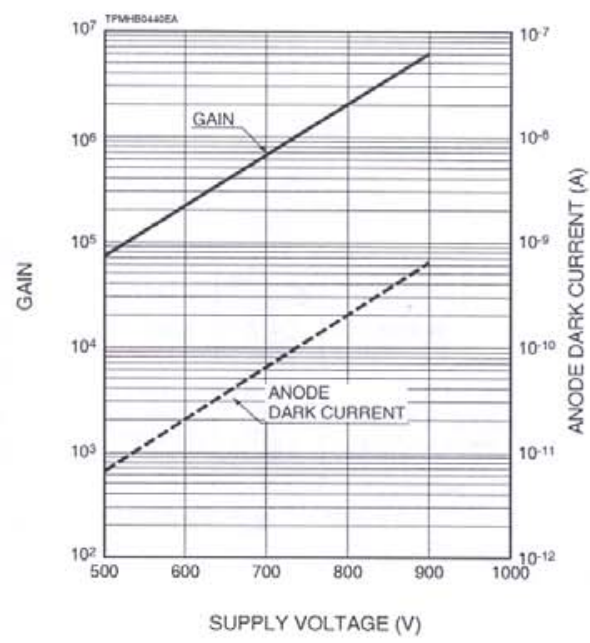

Figure 4: Typical Anode Uniformity

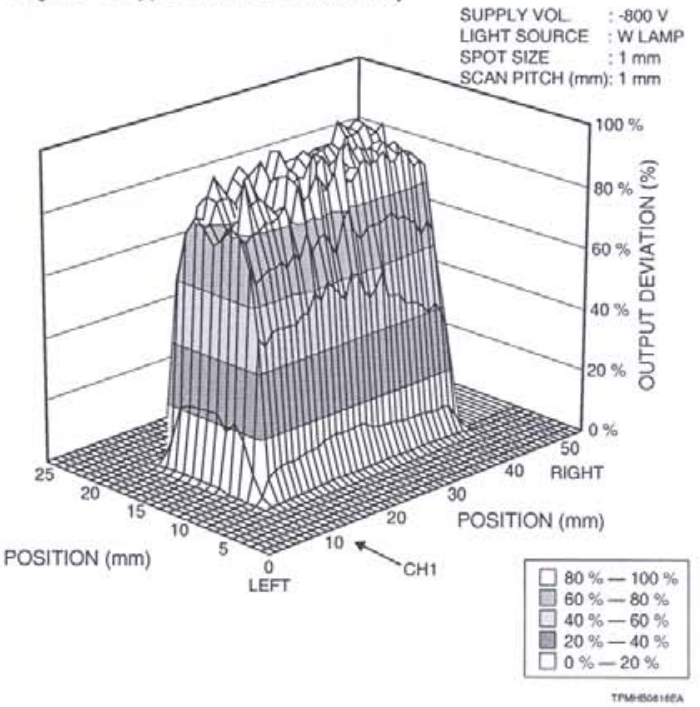

